\title{
Osteoconductive Microarchitecture of Bone Substitutes for Bone Regeneration Revisited
}

\author{
Chafik Ghayor ${ }^{1}$ and Franz E. Weber ${ }^{1,2,3 *}$ \\ 1 Oral Biotechnology and Bioengineering, Department of Cranio-Maxillofacial and Oral Surgery, Center for Dental Medicine, \\ University of Zurich, Zurich, Switzerland, ${ }^{2}$ Zurich Center for Integrative Human Physiology, University of Zurich, Zurich, \\ Switzerland, ${ }^{3}$ Center for Applied Biotechnology and Molecular Medicine, University of Zurich, Zurich, Switzerland
}

OPEN ACCESS

Edited by:

Gianpaolo Papaccio, Università degli Studi della Campania

"Luigi Vanvitelli" Naples, Italy

Reviewed by:

Virginia Tirino,

Università degli Studi della Campania

"Luigi Vanvitelli" Naples, Italy

Francesco Zotta,

Humanitas Università, Italy

${ }^{*}$ Correspondence:

Franz E. Weber

franz.weber@zzm.uzh.ch

Specialty section:

This article was submitted to Craniofacial Biology and Dental

Research,

a section of the journal

Frontiers in Physiology

Received: 24 April 2018

Accepted: 29 June 2018

Published: 19 July 2018

Citation:

Ghayor C and Weber FE (2018) Osteoconductive Microarchitecture of Bone Substitutes for Bone

Regeneration Revisited.

Front. Physiol. 9:960.

doi: 10.3389/fphys.2018.00960
In the last three decades, all efforts in bone tissue engineering were driven by the dogma that the ideal pore size in bone substitutes lies between 0.3 and $0.5 \mathrm{~mm}$ in diameter. Newly developed additive manufacturing methodologies for ceramics facilitate the total control over pore size, pore distribution, bottleneck size, and bottleneck distribution. Therefore, this appears to be the method of choice with which to test the aforementioned characteristics of an ideal bone substitute. To this end, we produced a library of 15 scaffolds with diverse defined pore/bottleneck dimensions and distributions, tested them in vivo in a calvarial bone defect model in rabbits, and assessed the clinically most relevant parameters: defect bridging and bony regenerated area. Our in vivo data revealed that the ideal pore/bottleneck dimension for bone substitutes is in the range of $0.7-1.2 \mathrm{~mm}$, and appears therefore to be twofold to fourfold more extended than previously thought. Pore/bottleneck dimensions of 1.5 and $1.7 \mathrm{~mm}$ perform significantly worse and appear unsuitable in bone substitutes. Thus, our results set the ideal range of pore/bottleneck dimensions and are likely to have a significant impact on the microarchitectural design of future bone substitutes for use in orthopedic, trauma, cranio-maxillofacial and oral surgery.

\footnotetext{
Keywords: osteoconduction, pore, bone substitute material, additive manufacturing, lithography, micro architecture, bone regeneration, scaffold
}

\section{INTRODUCTION}

Bones have the natural ability to heal due to the presence of osteoinductive proteins in the bone matrix (Urist, 1965) and mesenchymal progenitor and stem cells in the bone marrow, the periost and other surrounding tissues (Owen and Friedenstein, 1988; Bianco et al., 2008). If bone defects surpass a critical size, however, transplantation of autologous tissue is often a necessary requirement to aid the regenerative process. Bone is the second most frequently transplanted tissue in Europe after blood, with around one million procedures performed annually. The worldwide market of bone replacement material is currently estimated at five billion $€$ and is increasing by $10 \%$ every year (Medical Press, 2017). Importantly, the outcome of bone transplantation is not only dependent on the osteoinductive nature of the implanted material, but also on its osteoconductive properties.

Osteoconduction defines a three dimensional process observed when porous structures are implanted in or adjacent to bone. The porous spaces are initially infiltrated by capillaries, 
perivascular tissues, and osteoprogenitor cells, followed by incorporation of the porous structure within the newly formed bone (Cornell and Lane, 1998). Osteoconduction was first described by Barth (1893) following the histological analysis of the fate of transplanted autologous bone. Findings revealed that the transplanted bone was degraded and replaced by newly formed bone through a process termed creeping substitution (Axhausen, 1909). It has since been demonstrated that during the latter phases of this process, the degradation of the autologous bone liberates calcium phosphates and osteoinductive proteins (Cornell and Lane, 1998), which further serve to enhance bone regeneration Although considered the gold standard for bone repair, the harvesting of autologous bone carries with it the risk of donor site morbidity. This, together with limitations in its availability, have led investigators to seek out alternative bone substitutes, with the aim to developing off-the-shelf products for treating bone defects (Burchardt, 1983). In designing bone substitute, great emphasis is placed on material type, porosity and surface, all of which influence the efficiency of bone ingrowth. The influence of material surface in particular has been a primary focus in the development of dental implants where efficient osseointegration is critical (Buser et al., 1991). For titanium implants, the best surface appeared to be a moderately rough one (Schwartz et al., 1999; Wennerberg and Albrektsson, 2010), whereas for calcium phosphate-based bone substitutes, submicron surface structures outperformed larger micron scaled surfaces in terms of their ability to stimulate the osteogenic differentiation of multipotent stromal cells (Zhang et al., 2017a,b).

Over the last two decades, the field of bone regeneration advanced by the introduction of advanced medical technology and surgical procedures (Rullo et al., 2013), bone morphogenetic proteins and osteoinduction (Urist, 1965; Carragee et al., 2011; Martin et al., 2015) and by the application of research results from the mesenchymal stem cell field (Cancedda et al., 2003; Bianco et al., 2008; Mehrkens et al., 2012; Brennan et al., 2014; Paino et al., 2017) or the neural-crest derived stem cell field (d'Aquino et al., 2011; Spina et al., 2016; Gazarian and Ramírez-García, 2017). In exception of the application of more advanced medical technology or surgical procedures these strategies, however, are costly and associated with increased risks for the patient (Carragee et al., 2011). For the application of bone morphogenetic proteins and stem cells, a carrier system is needed. Ideally, such a delivery system is osteoconductive. In terms of osteoconductive microarchitecture it was shown that bone substitutes containing concave pits induce significantly more bone tissue formation than smooth surfaces (Graziano et al., 2007) and that bone formation benefits from concavities on the surface of calcium phosphate based bone substitutes (Ripamonti, 2017). Another important microarchitectural feature studied for a long time was the optimal pore diameter. As a result of these studies a bone substitute pore diameter of $0.3-0.5 \mathrm{~mm}$ has long been regarded as the optimal size for osteoconduction, enabling efficient Haversiantype (Kuboki et al., 2001) and trabecular (LeGeros, 2002) bone formation. There is also the suggestion that bone substitutes with pore sizes in excess of $0.4 \mathrm{~mm}$ are less conducive to new bone formation as evidenced by the accumulation of adipocytes and bone marrow (Tsuruga et al., 1997) and reduced mechanical properties (Kuboki et al., 2001). Optimal pore dimension of $0.2-$ $0.5 \mathrm{~mm}$ were also supported by several in vitro studies (reviewed in Perez and Mestres, 2016). Studies that are more recent reported on bone ingrowth and the presence of cells in micropores, well below $0.1 \mathrm{~mm}$ in diameter (Bernstein et al., 2013; Polak et al., 2013). There is only one in vivo study with random pore locations and undefined connections between pores suggesting that bone ingrowth is similar in pores from $0.5 \mathrm{~mm}$ up to $1.2 \mathrm{~mm}$ (von Doernberg et al., 2006). An upper limit in pore diameter for optimal bone ingrowth has not been determined yet.

The old dogma of the optimal mean pore size is mainly based on observations using scaffolds with single channels, or randomly distributed pores (Figure 1A). The emergence of additive manufacturing has since added a new dimension to the production of scaffolds, where pore size, as well as other microarchitectural constraints such as bottleneck dimensions can be accurately defined (Figures 1B,C). The term bottleneck dimension in this context is defined as the uniform diameter of the connections between pores and can be exactly tuned by additive manufacturing. In random pore distribution processes, however, the term percolation diameter was introduced (Ashworth et al., 2015) defined as the diameter of the largest tracer sphere able to move through a scaffold of interconnected pores and reflects the smallest diameter of a single connection in an interconnected pore system.

The effectiveness of $3 \mathrm{D}$ printing as an additive manufacturing technique in regenerative medicine, particularly bone tissue engineering, has been well reviewed (Hutmacher, 2000; Jariwala et al., 2015). Using this technology to define bone substitute microarchitecture is, however, still in its infancy (Seitz et al., 2005; Carrel et al., 2016), although the production of fine open structures composed of calcium phosphates is now possible using for example lithography-based additive manufacturing (CeraFab 7500, Lithoz, Vienna, Austria). The aim of this project was to design and produce a library of tricalcium phosphate-based scaffolds with defined pore sizes and bottleneck dimensions using lithography-based additive manufacturing, and to identify the most osteoconductive microarchitecture based on its potential to support defect bridging and new bone formation in vivo.

\section{MATERIALS AND METHODS}

\section{Design and Fabrication of Scaffolds}

We used the computer aided design software tool SolidWorks (Dassault Systèmes SolidWorks Corporation, Waltham, MA, United States) to design a library of 20 different stepped scaffolds with a diameter of $6 \mathrm{~mm}$ in the lower three levels, and $7.5 \mathrm{~mm}$ in the upper level as previously reported (de Wild et al., 2013) and illustrated in Figures 1C,D. The unit cells (Figure 1C) to design the scaffolds are cubes of 1.0-2.0 mm length. We have chosen this design and adjusted it to the needs of the material and production methodology, since it resembles the design with the highest mechanical performance and high anisotropy, as 
$\mathbf{A}$

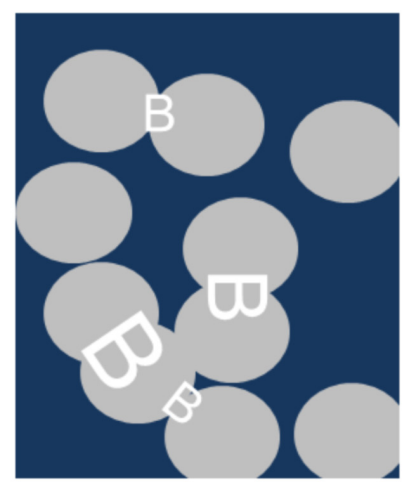

B

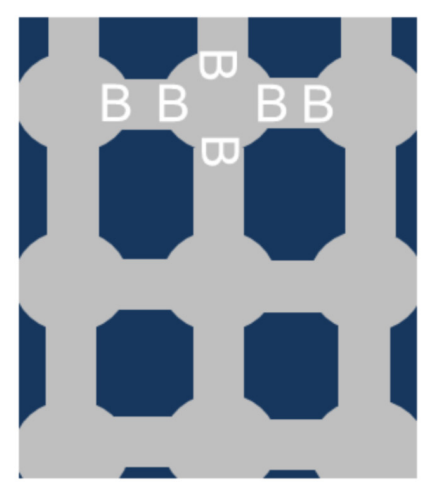

C
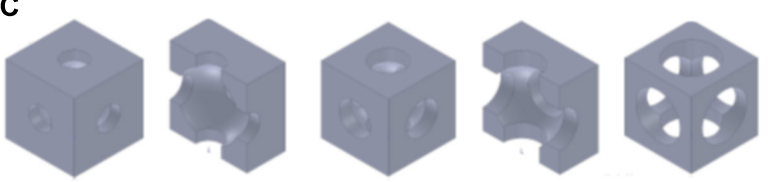

D

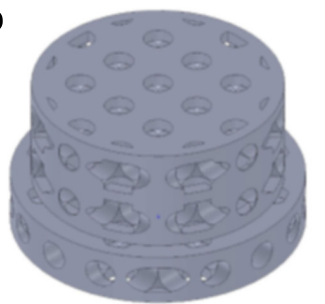

E

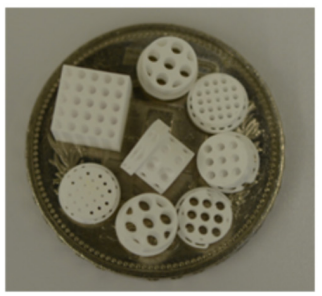

$\mathbf{F}$

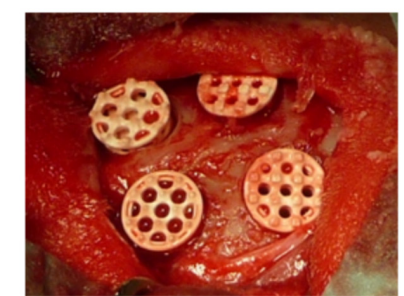

FIGURE 1 | Schematics and examples of bone substitute design, and in vivo testing of tri-calcium phosphate based scaffolds. Pore distribution and bottleneck dimension (B) are shown for porosogen based porous scaffolds. Scaffold in dark blue, and pores in gray (A). Pore distribution and bottleneck dimension (B) are shown for scaffolds produced by additive manufacturing. Scaffold in dark blue, and pores in gray (B). Example of unit cells as building block of the scaffolds with fixed cube and pore dimension but increase in bottleneck diameter from left to right. The unit cells views are provided in pairs: left: view on the unit cell, right: view on the halved unit cell (C). Design of one scaffold from the library (D). Scaffolds diverse in pore size and bottleneck dimension are displayed on a Swiss five-franc coin with a diameter of $32 \mathrm{~mm}(\mathbf{E})$. Intra operative view of scaffolds placed into four non-critical size defects of $6 \mathrm{~mm}$ in diameter created in the calvarial bone of a rabbit (F).

previously reported for titanium scaffolds (de Wild et al., 2016, 2018; Rüegg et al., 2017). In the center of each unit cell a pore of $0.5-1.7 \mathrm{~mm}$ is located. The pore of each unit cell is connected to all six sides of the cube with a central located cylinder with a diameter between 0.5 and $1.5 \mathrm{~mm}$. The latter diameter is equal to the bottleneck diameter. By the assembly of unit cells to form the scaffold the cylinders are connected and form an open path throughout the entire scaffold. All these parameters from scaffolds of our library are listed in Table 1. The fabrication of tri-calcium phosphate based scaffolds was performed using a CeraFab 7500 (Lithoz, Vienna, Austria). We used LithaBone TCP $200\left[\mathrm{Ca}_{3}\left(\mathrm{PO}_{4}\right)_{2}\right]$ as photosensitive slurry, consisting of tri-calcium phosphate powder of particle size in the range of 5$30 \mu \mathrm{m}$, and other undisclosed components like acrylate-based monomer, organic solvent, light absorber and photoinitiator. The CeraFab 7500 (Lithoz, Vienna, Austria) was used to solidify the slurry in a layer-by-layer fashion resulting in a green part with a resolution of $25 \mu \mathrm{m}$ in layer thickness, and $50 \mu \mathrm{m}$ in the $x / y$ plane. In the green part, the TCP particles are hold together by the polymer. Following its production, the green parts were removed from the building platform, cleaned from undetached slurry, and underwent a thermal treatment process to remove the solvent, to decompose the polymeric binder, and to sinter (densify) the samples. The program for thermal treatment was provided by the manufacturer, and included a final sintering step of $3 \mathrm{~h}$ at $1100^{\circ} \mathrm{C}$.

\section{Scaffold Characterization}

The numbers to describe the diverse scaffolds are displayed in Table 1. The porosity is the relative free volume describing the ratio of the material free volume inside the unit cell defined by the pore diameter and the diameter of the connections and the volume of the unit cell. The maximal transparency is the material free area in the projection of the unit cell in the spatial direction yielding the maximal value.

\section{Animal Experiments}

All animal procedures were approved by the Animal Ethics Committee of the local authorities (Canton Zurich, 108/2012 and 115/2015) and performed in accordance with the ethics criteria contained in the bylaws of the Institutional Animal Care and Use Committee. After the acclimatization period, implants were inserted into calvarial defects of 40 rabbits (female, 26week-old, New Zealand white rabbit), and bone regeneration determined after 4 weeks as previously described (KarfeldSulzer et al., 2014). In brief, animals were anesthetized by injection of $65 \mathrm{mg} / \mathrm{kg}$ ketamine and $4 \mathrm{mg} / \mathrm{kg}$ xylazine and 
TABLE 1 | Structural values for the different scaffolds.

\begin{tabular}{|c|c|c|c|c|c|c|}
\hline Acronym & $\begin{array}{l}\text { Length of unit } \\
\text { cell (mm) }\end{array}$ & $\begin{array}{l}\text { Pore diameter } \\
\qquad(\mathrm{mm})\end{array}$ & $\begin{array}{c}\text { Bottleneck } \\
\text { diameter }(\mathrm{mm})\end{array}$ & $\begin{array}{l}\text { Porosity } \\
\text { (Vol \%) }\end{array}$ & $\begin{array}{c}\text { Maximal } \\
\text { transparency } \\
\text { (surface \%) }\end{array}$ & $\begin{array}{c}\text { Surface area per } \\
\text { Vol }(1 / \mathrm{mm})\end{array}$ \\
\hline C_10_5_5 & 1.0 & 0.5 & 0.5 & 35.95 & 19.64 & 1.020 \\
\hline C_10_07_05 & 1.0 & 0.7 & 0.5 & 47.40 & 19.64 & 1.774 \\
\hline C_10_07_07 & 1.0 & 0.7 & 0.7 & 52.58 & 38.48 & 1.207 \\
\hline C_13_10_05 & 1.3 & 1.0 & 0.5 & 47.40 & 11.61 & 1.537 \\
\hline C_13_10_07 & 1.3 & 1.0 & 0.7 & 39.59 & 22.77 & 1.279 \\
\hline C_13_10_10 & 1.3 & 1.0 & 1.0 & 56.00 & 46.47 & 0.571 \\
\hline C_15_12_05 & 1.5 & 1.2 & 0.5 & 32.04 & 8.72 & 1.410 \\
\hline C_15_12_07 & 1.5 & 1.2 & 0.7 & 37.06 & 17.10 & 1.242 \\
\hline C_15_12_10 & 1.5 & 1.2 & 1.0 & 47.47 & 34.90 & 0.781 \\
\hline C_15_12_12 & 1.5 & 1.2 & 1.2 & 56,96 & 50.26 & 0.334 \\
\hline C_18_15_12 & 1.8 & 1.5 & 1.2 & 47.46 & 34.90 & 0.630 \\
\hline C_20_17_05 & 2.0 & 1.7 & 0.5 & 35.64 & 2.42 & 1.164 \\
\hline C_20_17_07 & 2.0 & 1.7 & 0.7 & 36.46 & 9.62 & 1.093 \\
\hline C_20_17_12 & 2.0 & 1.7 & 1.2 & 44.87 & 28.27 & 0.711 \\
\hline C_20_17_15 & 2.0 & 1.7 & 1.5 & 52.03 & 44.17 & 0.339 \\
\hline
\end{tabular}

further anesthetized with isofluoran $/ \mathrm{O}_{2}$. The surgical area was disinfected and an incision was made from the nasal bone to the midsagittal crest. Next, the soft tissues were reflected and the periosteum was elevated from the site. In the area of the right and left parietal and frontal bones, four evenly distributed $6 \mathrm{~mm}$ diameter craniotomy defects were prepared with a trephine bur under copious irrigation with sterile saline. For the completion of the defect a rose burr $(5 \mathrm{~mm})$ was used to preserve the dura. Before implant placement, bone debris were removed by flushing with saline. Each of the animals received four different treatment modalities. The treatment modalities were assigned at random for the first animal and thereafter cyclic permuted clockwise for the next three animals. Sample size was determined by power analysis.

\section{Histomorphometry}

The evaluation of all implants was performed from the MMAembedded middle section using image analysis software (ImagePro Plus ${ }^{\circledR}$; Media Cybernetic, Silver Springs, MD, United States). The area of interest (AOI) was defined by the $6 \mathrm{~mm}$ defect dimension and the height of the implant, corrected for differences in height between groups of different pore dimension. We determined the area of new bone in the AOI as percent of bone and bony integrated scaffold in the AOI (bony area, \%). For the empty control value, the average corrected area occupied by all scaffolds was taken into account.

\section{Bone Bridging}

The determination of bone bridging was performed as previously reported (Kruse et al., 2011; Schmidlin et al., 2013). In brief, areas with bone tissue were projected onto the $x$-axis. Next, the stretches of the $x$-axis where bone formation had occurred an an level were summed up and related to the defect width of $6 \mathrm{~mm}$. Bone bridging is given in percentage of the defect width $(6 \mathrm{~mm})$ where bone formation had occurred.

\section{Statistical Analysis}

The primary analysis unit was the animal. For all parameters tested, treatment modalities were compared with a KruskalWallis test, followed by Mann-Whitney signed rank test for independent data (IBM SPSS v.23). Significance was set at $P<0.05$. Values are reported as either mean \pm standard error, or displayed in box-plots ranging from the 25th (lower quartile) to the 75th (upper quartile) percentile including the median and whiskers showing the minimum and maximum values.

\section{RESULTS}

\section{Scaffolds With Pores Between 0.7 and $1.2 \mathrm{~mm}$ Are Optimal for Calvarial Bone Healing}

A total of 20 different scaffolds were prepared using the computer aided design software tool SolidWorks (Dassault Systèmes SolidWorks Corporation, Waltham, MA, United States), and their potential to support new bone growth assessed in a rabbit calvarial defect model (de Wild et al., 2013) (Figure 1E). Scaffolds were generated from the tri-calcium phosphate substrate Lithabone ${ }^{\mathrm{TM}}$ (Lithoz, Vienna, Austria) using the lithography-based additive manufacturing machine (CeraFab 7500, Lithoz, Vienna, Austria). Five scaffold designs failed mechanically during production, or during the in vivo testing stage, and are therefore not reported here. Following the removal of the photoactive binder, and sintering to increase mechanical stability all scaffolds exhibited a smooth surface with micropores of 2-4 $\mu \mathrm{m}$ (Chen et al. accepted Tissue Engineering). The so produced scaffolds, sterilized during the sintering procedure (Figure 1E) were transferred to the operation theater in a sterile fashion, implanted within the calvarial defects (Figure 1F), and bone formation assessed after 4 weeks. 


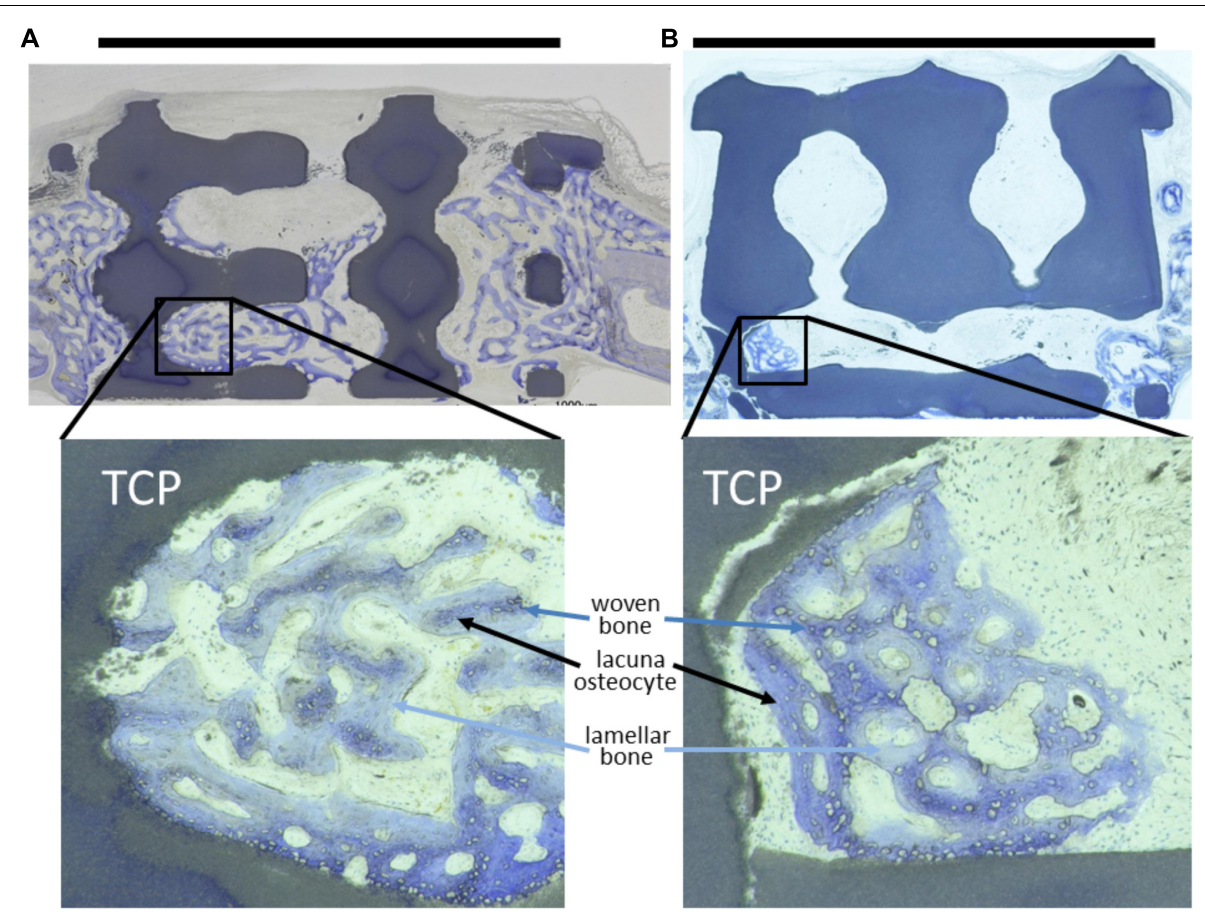

FIGURE 2 | Toluidine-blue stained MMA sections of middle sections from scaffold treated calvarial bone defects. Upon a healing period of 4 weeks, the scaffolds from tricalcium phosphate (TCP) are visible as blackish or dark blue areas. Bone ingrowth was most advanced in additively manufactured scaffolds with a pore diameter of $1.2 \mathrm{~mm}$ and bottleneck diameter of $1.0 \mathrm{~mm}$ (A). In scaffolds with a pore diameter of $1.5 \mathrm{~mm}$ and bottleneck diameter of $1.0 \mathrm{~mm}$ (B) bone growth did not extend far into the scaffolds. A scale bar, placed on top of the histologies is provided to show the 6 mm defect margins. In the lower panel, 2.5 -fold higher magnifications are shown from the area marked in (A,B) to visualize bone tissue formation. The light blue-stained bone tissue is lamellar bone (also new, but later formed) on the initial woven bone structures stained dark blue and purple. Lacunae from osteocytes are visible.

Histological analysis of methyl methacrylate (MMA)embedded tissue sections revealed predominantly woven bone formation in and around the scaffolds indicating good biocompatibility by new bone formation in close proximity to the scaffold material (Figure 2). Evaluation of osteoconduction was based on the level of bony bridging and bony regenerated area as determined by toluidine-blue staining. As compared to untreated empty defects, defects containing scaffolds with pore diameters of $1.2 \mathrm{~mm}$ and below performed better in terms of bony bridging and/or bony regeneration than scaffolds with a pore diameter of $1.5 \mathrm{~mm}$ or $1.7 \mathrm{~mm}$.

Quantitative analysis of the middle sections further confirmed that the percentage of bony bridging compared to untreated control defects was significantly greater in animals treated with scaffolds with pore diameters of $0.7,1.0,1.2,1.5$, and $1.7 \mathrm{~mm}$. Furthermore, scaffolds with pore sizes of $1.0 \mathrm{~mm}$ and bottlenecks of 0.7 and $1.0 \mathrm{~mm}$ and pore size of $1.2 \mathrm{~mm}$ and bottlenecks of $0.7,1.0$, and $1.2 \mathrm{~mm}$ proved significantly better as compared to scaffolds with pores sizes of 1.5 and $1.7 \mathrm{~mm}$, and bottlenecks of 0.7 and $1.2 \mathrm{~mm}$, respectively (Figure 3). Pore diameters of 1.5 and $1.7 \mathrm{~mm}$ were therefore considered the least beneficial for bony bridging.

The optimal pore diameter and bottleneck dimension for an osteoconductive scaffold is between 0.7 and $1.2 \mathrm{~mm}$ and below $1.5 \mathrm{~mm}$.
We next evaluated bony bridging and bony regenerated area dependency, grouped by pore diameter and bottleneck dimension. We found bony bridging of the defect to be significantly more complete in scaffolds with pores of 0.7 to $1.2 \mathrm{~mm}$ in diameter as compared to a pore diameter of $1.5 \mathrm{~mm}$ or $1.7 \mathrm{~mm}$ (Figure 4A). For the percentage of bony regenerated area in the defect, a pore diameter of $0.7-1.2 \mathrm{~mm}$ was significantly superior to a pore diameter of $1.5 \mathrm{~mm}$ (Figure 4C).

The percentage of bony bridging of the defect and the percentage of bony regenerated area of the defect of scaffolds with $0.5 \mathrm{~mm}$ pores were always in the range of scaffolds with $1.5 \mathrm{~mm}$ diameter pores and below scaffolds with pores of 0.7 , 1.0 , or $1.2 \mathrm{~mm}$ in diameter. Therefore, optimal pore diameter for both measures of osteoconductivity lies between 0.7 and $1.2 \mathrm{~mm}$. If the bottleneck between pores is between 0.5 and $1.2 \mathrm{~mm}$, bony bridging is significantly higher than for bottlenecks of $1.5 \mathrm{~mm}$ in diameter (Figure 4B). For bony regenerated area, only a bottleneck dimension of $1.2 \mathrm{~mm}$ was significant higher than one of $1.5 \mathrm{~mm}$ (Figure 4D). Grouping defect bridging according to porosity or transparency (Table 1) did not yield in any significant differences. The same applies to surface area per volume. Therefore, in this library of scaffolds from the identical material and identical surface structure pore diameter and bottleneck dimension were the key parameters of the microarchitecture to affect bony bridging and bony regeneration as measures for osteoconduction. 


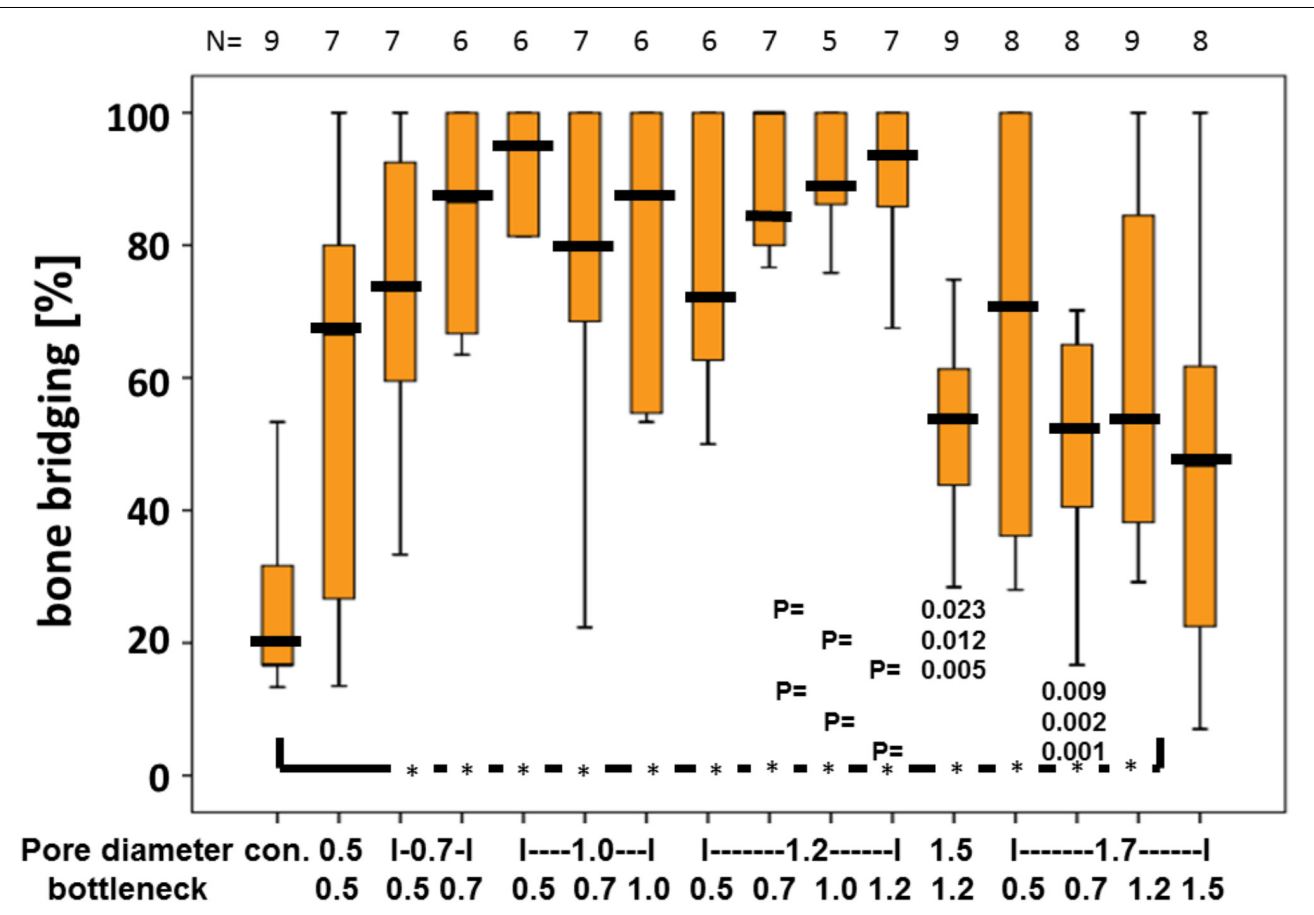

FIGURE 3 | Percentage of bony bridging of the defect in relation to pore and bottleneck dimensions. In comparison to the empty control, scaffolds with pore diameters from 0.7 to $1.7 \mathrm{~mm}$ and bottlenecks below $1.5 \mathrm{~mm}$ perform significantly better. Scaffolds with pores of $1.2 \mathrm{~mm}$ and bottlenecks between 0.7 and $1.2 \mathrm{~mm}$ perform significantly better than scaffolds with a pore diameter of $1.5 \mathrm{~mm}$ and bottleneck of $1.2 \mathrm{~mm}$ and pore diameter of $1.7 \mathrm{~mm}$ and bottleneck of $0.7 \mathrm{~mm}$. Results of each group are displayed as box-plots ranging from the 25th (lower quartile) to the 75th (upper quartile) percentile including the median as black bars and whiskers showing the minimum and maximum values. The number of samples for each group ( $\mathrm{N}$ ) is displayed on top of the figure.

\section{DISCUSSION}

In the current report, we generated a structurally diverse library of tri-calcium phosphate based scaffolds using $3 \mathrm{D}$ printing, and assessed their potential to influence bone formation in vivo. The osteoconductive capacity of bone implants is reliant on a complex interplay between the material type, surface and microarchitecture, which ultimately determines the efficiency of new bone formation and its vascularization. Here, we kept the material and surface characteristics constant and varied only the microarchitecture. The primary focus of our study was to challenge the long-held belief that optimal osteoconduction is achieved using bone substitutes with pore diameters of 0.3-0.5 mm (Tsuruga et al., 1997; Langer and Vacanti, 1999; Hutmacher, 2000; Galois and Mainard, 2004; Hollister, 2005; Murphy et al., 2010; Hollister and Murphy, 2011; Henkel et al., 2013) thereby opening up new possibilities for the improvement of scaffold microarchitecture design.

The results of our study demonstrated osteoconduction was significantly improved in bone substitutes with a pore diameter of 0.7-1.2 $\mathrm{mm}$ - an increase of up to fourfold above what the majority of published studies and reviews recommend. These findings are likely to pave the way for future developments in scaffold design, leading to the generation of bone substitutes with a more osteoconductive microarchitecture, and improved bone-regenerative capability (Figures $4 \mathbf{E}, \mathbf{F}$ ). Importantly, a pore size of $1.5 \mathrm{~mm}$ or greater had a detrimental effect on the bone bridging capabilities of the tri-calcium phosphate scaffolds used in this study; a notable diagnostic feature of non-unions. Given the clinical and economic burden of treating non-unions (Zura et al., 2016), there is an obvious need to develop bone substitutes with high osteoconductive properties.

Moreover, these data corroborate our previous observations on the influence of pore size on osteoconduction of titanium scaffolds (de Wild et al., 2013, 2016, 2018). Our results are in line with an in vivo sheep study reporting on drill hole defects in cancellous bone where scaffolds with random distributed pore sizes of $0.15,0.26,0.51$, and $1.22 \mathrm{~mm}$ but undefined pore location and bottleneck dimension or percolation were tested (von Doernberg et al., 2006). In terms of bone regeneration, they did not see huge differences. It should be noted, however, that due to technical restraints of our additive manufacturing process, generating bone substitutes with pore sizes below $0.5 \mathrm{~mm}$ was not technically possible $-\mathrm{n}$ obvious limitation of this system. Importantly, a pore size of 1.5 or $1.7 \mathrm{~mm}$ or a bottleneck of $1.5 \mathrm{~mm}$ had a detrimental effect on the bone bridging and bony regeneration capabilities of the tri-calcium phosphate scaffolds used in our study. This sets a so far unknown upper limit to osteoconductive pore sizes at $1.2 \mathrm{~mm}$ and below $1.5 \mathrm{~mm}$.

For polycaprolactone based scaffolds, a more permeable scaffold with regular architecture performed best for in vivo 

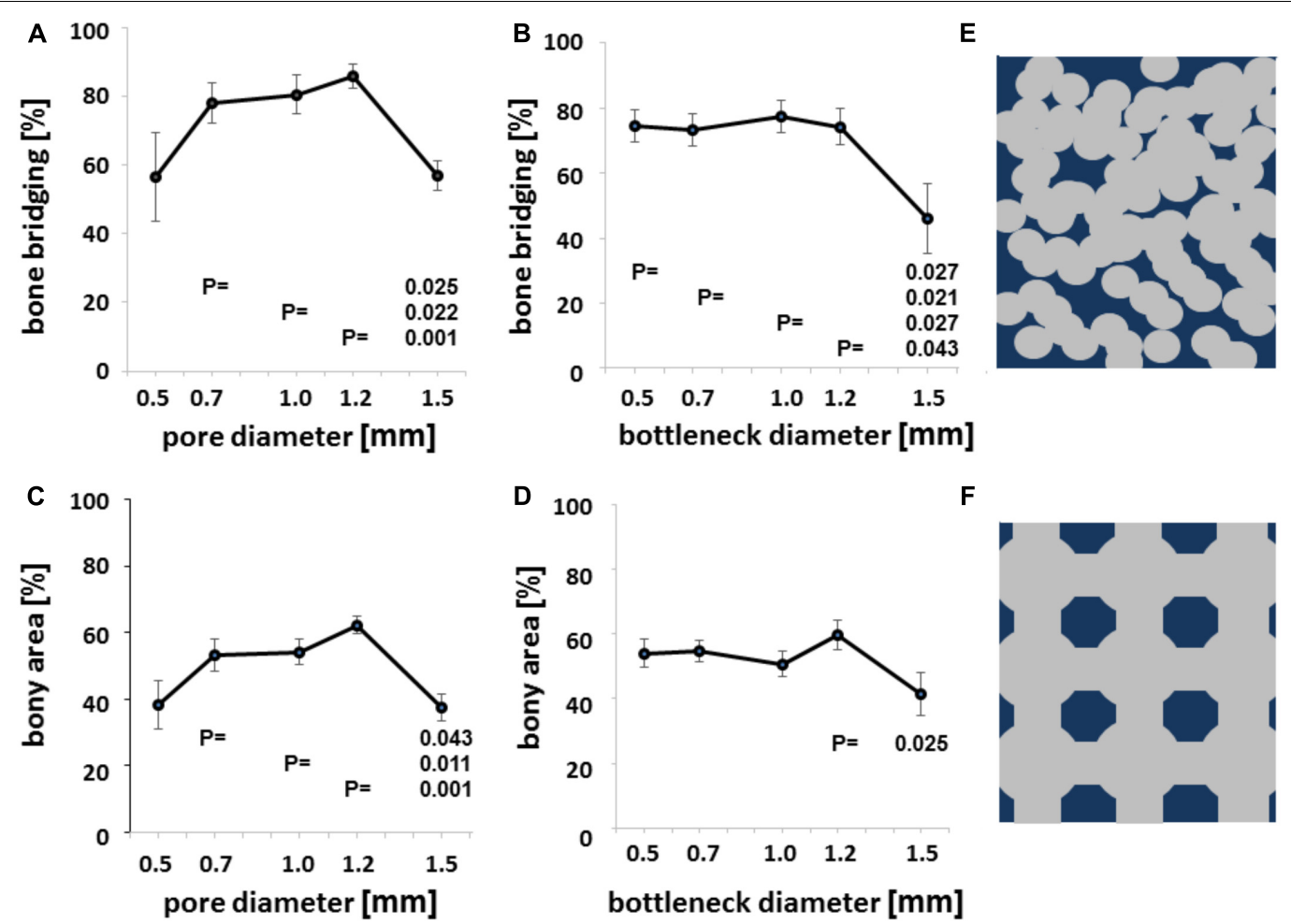

FIGURE 4 | Microarchitecture and osteoconduction. Bony bridging in relation to pore diameter of all groups (A). Bony bridging and bottleneck diameter. Bony bridging in relation to bottleneck diameter of all groups (B). Bony regenerated area and pore diameter. Bony regenerated area in relation to bottleneck diameter of all groups (C). Bony regenerated area and bottleneck diameter. Bony regenerated area in relation to bottleneck diameter of all groups (D). All graphs display the means and the standard error of the means. P-values are displayed. Schematic drawing of conventional scaffold based on random distributed $0.5 \mathrm{~mm}$ diameter pores. Scaffold in dark blue and pores in gray (E). Schematic drawing of additively manufactured scaffold based on $1.2 \mathrm{~mm}$ pores and bottlenecks of $0.7 \mathrm{~mm}$ Scaffold in dark blue and pores in gray (F).

bone regeneration (Mitsak et al., 2011). The microarchitecture we have tested here is not only permeable but also transparent from each plane of the cube of the unit cell throughout the entire scaffold, since all empty cylinders connecting the pores are aligned. As listed in Table 1, the transparency of unit cells $1.5 \mathrm{~mm}$ in length and a pore diameter of $1.2 \mathrm{~mm}$ increases with the bottleneck diameter $(0.5-1.2 \mathrm{~mm})$ from 8.72 to $50.26 \%$. Bony bridging for those scaffolds follow the same trend, but fail to differ significantly. The same applies to porosity, which also increases from 32.04 to $56.96 \%$. Scaffolds with pores of $1.5 \mathrm{~mm}$ perform significantly worse in terms of bony bridging, despite the fact that with a transparency of $34.90 \%$ and a porosity of $47.46 \%$ both characteristics are more at the higher end. Moreover, the surface area per volume of scaffolds derived from the unit cell of $1.5 \mathrm{~mm}$ in length and a pore diameter of $1.2 \mathrm{~mm}$ decreases with the increase in bottleneck diameter (0.5-1.2 $\mathrm{mm}$ from 1.41 to $0.331 / \mathrm{mm}$ (Table 1). Therefore, bony bridging and surface area follow opposite trends. Taken together, with our microarchitecture bony bridging follows permeability and porosity in groups derived from the same unit cell. The most important factor, however, appears to be pore diameter. To fully understand all these relations, additional research with more designs and additional model systems are needed.
Interestingly, it was proposed that in the initial weeks bone regeneration depends mainly on material aspects and that design aspects come in play only at later stages (Kommareddy et al., 2010; Tamjid et al., 2013). This is true for in vitro situations. In vivo, however, we show that with a constant material and surface structure, osteoconduction even during the first 4 weeks depends heavily on pore size and bottleneck dimension and therefore on microarchitectural features. That in vitro and in vivo results on bone tissue engineering approaches can contradict each other has been noted by others as well (Karageorgiou and Kaplan, 2005).

Autologous bone is still the bone substitute material of choice for treating critical size defects (De Long et al., 2007). Since the porosity of trabecular bone is between 0.2 and $0.4 \mathrm{~mm}$ (LeGeros, 2002), it was reasonable to assume that scaffolds with comparable pore dimensions would provide a more physiologically relevant bone substitute. In living bone, however, the microarchitecture reflects the local mechanical needs (Keaveny et al., 2001) and thus, no evolutionary pressure exists on osteoconduction in terms of bone ingrowth into 3D-structures. One can speculate that the optimal pore and bottleneck dimensions derived from this study reflect the balance between the positive interactions of directionally growing bone tissue with the scaffold as guiding cue, and the restrictions imposed by the scaffold on directional bone 
growth. Clearly, this balance is tipped in favor of the scaffold's positive effects, as made evident by the significant improvement in bony bridging when using bone substitutes with pore sizes between 0.7 and $1.2 \mathrm{~mm}$, and bottlenecks between 0.5 and $1.2 \mathrm{~mm}$ instead of pores and bottlenecks of $1.5 \mathrm{~mm}$ and more (Figure 3).

Our scaffolds consisted of tri-calcium phosphate, which is often used for scaffolds in bone tissue engineering as it degrades faster than native hydroxyapatite, whilst remaining biodegradable even after sintering at temperatures above $1,100^{\circ} \mathrm{C}$ (Goto et al., 2001). However, in the current study, scaffold biodegradation was not taken into consideration based on the fact that it takes several months for tri-calcium phosphate to completely be removed from the defect site (Walsh et al., 2008).

Additive manufacturing of free form scaffolds alleviated some of the constraints of extrusion-based techniques, such as the filament dimension ruling the pore dimension in the $z$-axis and the mechanics of the filament the pore dimension in the $x$ - to $y$-axis. In the past, porogens were considered a necessary component of the porous bone substitute production process, but led to random pore distribution, uncontrollable bottleneck dimensions, and restricted research of scaffolds microarchitecture (Figure 4E). We envisage that the use of additively manufactured bone substitutes with pore diameters in the range of $0.7-1.2 \mathrm{~mm}$, and a bottleneck dimension of $0.5-1.2 \mathrm{~mm}$, offer the best solution achieving optimal bone regeneration, and have the potential to revolutionize the way we treat bone defects.

\section{CONCLUSION}

The microarchitecture of bone substitutes based on pores and bottlenecks is most osteoconductive with pore diameters

\section{REFERENCES}

Ashworth, J. C., Mehr, M., Buxton, P. G., Best, S. M., and Cameron, R. E. (2015). Cell invasion in collagen scaffold architectures characterized by percolation theory. Adv. Healthc. Mater. 4, 1317-1321. doi: 10.1002/adhm.2015 00197

Axhausen, G. (1909). Histologische untersuchungen über knochentransplantation am menschen. Dtsch. Z. Chir. 91, 388-428. doi: 10.1007/BF02816571

Barth, A. (1893). Ueber histologische befunde nach knochenimplantationen. Arch. Klin. Chir. 46, 409-417.

Bernstein, A., Niemeyer, P., Salzmann, G., Südkamp, N. P., Hube, R., Klehm, J., et al. (2013). Microporous calcium phosphate ceramics as tissue engineering scaffolds for the repair of osteochondral defects: histological results. Acta Biomater. 9, 7490-7505. doi: 10.1016/j.actbio.2013.03.021

Bianco, P., Robey, P. G., and Simmons, P. J. (2008). Mesenchymal stem cells: revisiting history, concepts, and assays. Cell Stem Cell 2, 313-319. doi: 10.1016/ j.stem.2008.03.002

Brennan, M., Renaud, A., Amiaud, J., Rojewski, M., Schrezenmeier, H., Heymann, D., et al. (2014). Pre-clinical studies of bone regeneration with human bone marrow stromal cells and biphasic calcium phosphate. Stem Cell Res. Ther. 5:114. doi: 10.1186/scrt504

Burchardt, H. (1983). The biology of bone graft repair. Clin. Orthop. Relat. Res. 174, 28-42. doi: 10.1097/00003086-198304000-00005

Buser, D., Schenk, R. K., Steinemann, S., Fiorellini, J. P., Fox, C. H., and Stich, H. (1991). Influence of surface characteristics on bone integration of titanium implants. A histomorphometric study in miniature pigs. J. Biomed. Mater. Res. 25, 889-902. doi: 10.1002/jbm.820250708 between 0.7 and $1.2 \mathrm{~mm}$ and bottlenecks between 0.5 and $1.2 \mathrm{~mm}$. Pores and bottlenecks of $1.5 \mathrm{~mm}$ and beyond are detrimental for osteoconduction. In order to generate such microarchitecture, additive manufacturing is likely to become a central player, enabling the production of reproducible osteoconductive microarchitectures, which can be adjusted according to mechanical needs. Furthermore, additive manufacturing will be an invaluable tool in developing strategies geared toward personalized treatment, where the generation of scaffolds with patient specific bone defect dimensions is highly desirable.

\section{AUTHOR CONTRIBUTIONS}

FW designed the experiments. CG and FW performed the experiments, analyzed the data, and wrote the manuscript.

\section{FUNDING}

This research work was supported by grants from the Swiss National Science Foundation to FW.

\section{ACKNOWLEDGMENTS}

We thank Ana Perez, Indranil Bhattacharya, and Alexandre Tchouboukov for excellent technical assistance. All animal work was kindly supported by Flora Nichols and Thea Fleischmann. We are grateful to Peter J. Richards for editing the final version of the manuscript.

Cancedda, R., Dozin, B., Giannoni, P., and Quarto, R. (2003). Tissue engineering and cell therapy of cartilage and bone. Matrix Biol. 22, 81-91. doi: 10.1016/ S0945-053X(03)00012-X

Carragee, E. J., Hurwitz, E. L., and Weiner, B. K. (2011). A critical review of recombinant human bone morphogenetic protein-2 trials in spinal surgery: emerging safety concerns and lessons learned. Spine J. 11, 471-491. doi: 10. 1016/j.spinee.2011.04.023

Carrel, J. P., Wiskott, A., Moussa, M., Rieder, P., Scherrer, S., and Durual, S. (2016). A 3D printed TCP/HA structure as a new osteoconductive scaffold for vertical bone augmentation. Clin. Oral. Implants Res. 27, 55-62. doi: 10.1111/clr.12503

Cornell, C. N., and Lane, J. M. (1998). Current understanding of osteoconduction in bone regeneration. Clin. Orthop. Relat. Res. 355, S267-S273. doi: 10.1097/ 00003086-199810001-00027

d'Aquino, R., Tirino, V., Desiderio, V., Studer, M., De Angelis, G., Laino, C. L., et al. (2011). Human neural crest-derived postnatal cells exhibit remarkable embryonic attributes either in vitro or in vivo. Eur. Cell Mater. 21, 304-316. doi: 10.22203/eCM.v021a23

De Long, W. G. J., Einhorn, T. A., Koval, K., McKee, M., Smith, W., Sanders, R., et al. (2007). Bone grafts and bone graft substitutes in orthopaedic trauma surgery: a critical analysis. J. Bone Joint Surg. Am. 89, 649-658. doi: 10.2106/ JBJS.F.00465

de Wild, M., Ghayor, C., Zimmermann, S., Rüegg, J., Nicholls, F., Schuler, F., et al. (2018). Osteoconductive lattice microarchitecture for optimized bone regeneration. J. 3D Print. Addit. Manuf. doi: 10.1089/3dp.2017.0129 [Epub ahead of print].

de Wild, M., Schumacher, R., Mayer, K., Schkommodau, E., Thoma, D. S., Bredell, M., et al. (2013). Bone regeneration by the osteoconductivity of porous 
titanium implants manufactured by selective laser melting: a histological and $\mu \mathrm{CT}$ study in the rabbit. Tissue Eng. Part A 19, 2645-2654. doi: 10.1089/ten. TEA.2012.0753

de Wild, M., Zimmermann, S., Rüegg, J., Schumacher, R., Fleischmann, T., Ghayor, C., et al. (2016). Influence of microarchitecture on osteoconduction and mechanics of porous titanium scaffolds generated by selective laser melting. J. 3D Print. Addit. Manuf. 3, 142-151. doi: 10.1089/3dp.2016.0004

Galois, L., and Mainard, D. (2004). Bone ingrowth into two porous ceramics with different pore sizes: an experimental study. Acta Orthop. Belg. 70, 598-603.

Gazarian, K. G., and Ramírez-García, L. R. (2017). Human deciduous teeth stem cells (SHED) display neural crest signature characters. PLoS One 12:e170321. doi: 10.1371/journal.pone.0170321

Goto, T., Kojima, T., Iijima, T., Yokokura, S., Kawano, H., Yamamoto, A., et al. (2001). Resorption of synthetic porous hydroxyapatite and replacement by newly formed bone. J. Orthop. Sci. 6, 444-447. doi: 10.1007/s007760170013

Graziano, A., d'Aquino, R., Cusella-De Angelis, M. G., Laino, G., Piattelli, A., Pacifici, M., et al. (2007). Concave pit-containing scaffold surfaces improve stem cell-derived osteoblast performance and lead to significant bone tissue formation. PLoS One 2:e496. doi: 10.1371/journal.pone.0000496

Henkel, J., Woodruff, M. A., Epari, D. R., Steck, R., Glatt, V., Dickinson, I. C., et al. (2013). Bone regeneration based on tissue engineering conceptions a 21st century perspective. Bone Res. 1, 216-248. doi: 10.4248/BR201 303002

Hollister, S. J. (2005). Porous scaffold design for tissue engineering. Nat. Mater. 4, 518-524. doi: 10.1038/nmat1421

Hollister, S. J., and Murphy, W. L. (2011). Scaffold translation: barriers between concept and clinic. Tissue Eng. Part B Rev. 17, 459-474. doi: 10.1089/ten.TEB. 2011.0251

Hutmacher, D. W. (2000). Scaffolds in tissue engineering bone and cartilage. Biomaterials 21, 2529-2543. doi: 10.1016/S0142-9612(00)00121-6

Jariwala, S. H., Lewis, G. S., Bushman, Z. J., and Adair, J. H. (2015). 3D printing of personalized artificial bone scaffolds. 3D Print. Addit. Manuf. 2, 56-64. doi: $10.1089 / 3 \mathrm{dp} .2015 .0001$

Karageorgiou, V., and Kaplan, D. (2005). Porosity of 3D biomaterial scaffolds and osteogenesis. Biomaterials 26, 5474-5491. doi: 10.1016/j.biomaterials. 2005 . 02.002

Karfeld-Sulzer, L. S., Ghayor, C., Siegenthaler, B., Gjoksi, B., Pohjonen, T. H., and Weber, F. E. (2014)). Comparative study of NMP-preloaded and dip-loaded membranes for guided bone regeneration of rabbit cranial defects. J. Tissue Eng. Regen. Med. 11, 425-433. doi: 10.1002/term.1926

Keaveny, T. M., Morgan, E. F., Niebur, G. L., and Yeh, O. C. (2001). Biomechanics of trabecular bone. Annu. Rev. Biomed. Eng. 3, 307-333. doi: 10.1146/annurev. bioeng.3.1.307

Kommareddy, K. P., Lange, C., Rumpler, M., Dunlop, J., Manjubala, W. C. I., Cui, J., et al. (2010). Two stages in three-dimensional in vitro growth of tissue generated by osteoblastlike cells. Biointerphases 5, 45-52. doi: 10.1116/ 1.3431524

Kruse, A., Jung, R. E., Nicholls, F., Zwahlen, R. A., Hammerle, C. H., and Weber, F. E. (2011). Bone regeneration in the presence of a synthetic hydroxyapatite/silica oxide-based and a xenogenic hydroxyapatite-based bone substitute material. Clin. Oral. Implants. Res. 22, 506-511. doi: 10.1111/j.16000501.2010.02039.x

Kuboki, Y., Jin, Q., and Takita, H. (2001). Geometry of carriers controlling phenotypic expression in bmp-induced osteogenesis and chondrogenesis. J. Bone Joint Surg. Am. 83, S105-S115. doi: 10.2106/00004623-20010000200005

Langer, R. S., and Vacanti, J. P. (1999). Tissue engineering: the challenges ahead. Sci. Am. 280, 86-89. doi: 10.1038/scientificamerican0499-86

LeGeros, R. Z. (2002). Properties of osteoconductive biomaterials: calcium phosphates. Clin. Orthop. Relat. Res. 395, 81-98. doi: 10.1097/00003086200202000-00009

Martin, B. I, Lurie, J. D., Tosteson, A. N., Deyo, R. A., Farrokhi, F. R., and Mirza, S. K. (2015). Use of bone morphogenetic protein among patients undergoing fusion for degenerative diagnoses in the United States, 2002 to 2012. Spine J. 15, 692-699. doi: 10.1016/j.spinee.2014.12.010

Medical Press (2017). Growing New Bone for More Effective Injury Repair. Available at: https://medicalxpress.com/news/2017-03-bone-effective-injury.html
Mehrkens, A., Saxer, F., Guven, S., Hoffmann, W., Muller, A. M., Jakob, M., et al. (2012). Intraoperative engineering of osteogenic grafts combining freshly harvested, human adipose-derived cells and physiological doses of bone morphogenetic protein-2. Eur. Cell Mater. 24, 308-319. doi: 10.22203/eCM. v024a 22

Mitsak, A. G., Kemppainen, J. M., Harris, M. T., and Hollister, S. J. (2011). Effect of polycaprolactone scaffold permeability on bone regeneration in vivo. Tissue Eng. Part A 17, 1831-1839. doi: 10.1089/ten.TEA.2010.0560

Murphy, C. M., Haugh, M. G., and OBrien, F. J. (2010). The effect of mean pore size on cell attachment, proliferation and migration in collagen-glycosaminoglycan scaffolds for bone tissue engineering. Biomaterials 31, 461-466. doi: 10.1016/j. biomaterials.2009.09.063

Owen, M., and Friedenstein, A. J. (1988). Stromal stem cells: marrow-derived osteogenic precursors. Ciba Found. Symp. 136, 42-60.

Paino, F., La Noce, M., Giuliani, A., De Rosa, A., Mazzoni, S., Laino, L., et al. (2017). Human DPSCs fabricate vascularized woven bone tissue: a new tool in bone tissue engineering. Clin. Sci. 131, 699-713. doi: 10.1042/CS2017 0047

Perez, R. A., and Mestres, G. (2016). Role of pore size and morphology in musculoskeletal tissue regeneration. Mater. Sci. Eng. C Mater. Biol. Appl. 61, 922-939. doi: 10.1016/j.msec.2015.12.087

Polak, S. J., Rustom, L. E., Genin, G. M., Talcott, M., and Wagoner Johnson, A. J. (2013). A mechanism for effective cell-seeding in rigid, microporous substrates. Acta Biomater. 9, 7977-7986. doi: 10.1016/j.actbio.2013. 04.040

Ripamonti, U. (2017). Functionalized surface geometries induce: bone: formation by autoinduction. Front. Physiol. 8:1084. doi: 10.3389/fphys.2017.01084

Rüegg, J., Schumacher, R., Weber, F. E., and de Wild, M. (2017). Mechanical anisotropy of titanium scaffolds. Curr. Dir. Biomed. Eng. 3, 607-611. doi: 10. 1016/j.actbio.2009.11.032

Rullo, R., Addabbo, F., Papaccio, G., DAquino, R., and Festa, V. M. (2013). Piezoelectric device vs. conventional rotative instruments in impacted third molar surgery: relationships between surgical difficulty and postoperative pain with histological evaluations. J. Craniomaxillofac. Surg. 41, e33-e38. doi: 10. 1016/j.jcms.2012.07.007

Schmidlin, P. R., Nicholls, F., Kruse, A., Zwahlen, R. A., and Weber, F. E. (2013). Evaluation of moldable, in situ hardening calcium phosphate bone graft substitutes. Clin. Oral. Implants Res. 24, 149-157. doi: 10.1111/j.1600-0501. 2011.02315.x

Schwartz, Z., Lohmann, C. H., Oefinger, J., Bonewald, L. F., Dean, D. D., and Boyan, B. D. (1999). Implant surface characteristics modulate differentiation behavior of cells in the osteoblastic lineage. Adv. Dent. Res. 13, 38-48. doi: 10.1177/08959374990130011301

Seitz, H., Rieder, W., Irsen, S., Leukers, B., and Tille, C. (2005). Three-dimensional printing of porous ceramic scaffolds for bone tissue engineering. J. Biomed. Mater. Res. B Appl. Biomater. 74, 782-788. doi: 10.1002/jbm.b.30291

Spina, A., Montella, R., Liccardo, D., De Rosa, A., Laino, L., Mitsiadis, T. A., et al. (2016). NZ-GMP approved serum improve hDPSC osteogenic commitment and increase angiogenic factor expression. Front. Physiol. 7:354. doi: 10.3389/ fphys.2016.00354

Tamjid, E., Simchi, A., Dunlop, J. W. C., Fratzl, P., Bagheri, R., and Vossoughi, M. (2013). Tissue growth into three-dimensional composite scaffolds with controlled micro-features and nanotopographical surfaces. J. Biomed. Mater. Res. A 101, 2796-2807. doi: 10.1002/jbm.a.34584

Tsuruga, E., Takita, H., Itoh, H., Wakisaka, Y., and Kuboki, Y. (1997). Pore size of porous hydroxyapatite as the cell-substratum controls bmp-induced osteogenesis. J. Biochem. 121, 317-324. doi: 10.1093/oxfordjournals.jbchem. a021589

Urist, M. R. (1965). Bone: formation by autoinduction. Science 150, 893-899. doi: $10.1126 /$ science.150.3698.893

von Doernberg, M.-C., von Rechenberg, B., Bohner, M., Grünenfelder, S., van Lenthe, G. H., Müller, R., et al. (2006). In vivo behavior of calcium phosphate scaffolds with four different pore sizes. Biomaterials 27, 5186-5198. doi: 10. 1016/j.biomaterials.2006.05.051

Walsh, W. R., Vizesi, F., Michael, D., Auld, J., Langdown, A., Oliver, R., et al. (2008). Beta-TCP bone graft substitutes in a bilateral rabbit tibial defect model. Biomaterials 29, 266-271. doi: 10.1016/j.biomaterials.2007. 09.035 
Wennerberg, A., and Albrektsson, T. (2010). On implant surfaces: a review of current knowledge and opinions. Int. J. Oral Maxillofac. Implants 25, 63-74.

Zhang, J., Dalbay, M. T., Luo, X., Vrij, E., Barbieri, D., Moroni, L., et al. (2017a). Topography of calcium phosphate ceramics regulates primary cilia length and TGF receptor recruitment associated with osteogenesis. Acta Biomater. 57, 487-497. doi: 10.1016/j.actbio.2017. 04.004

Zhang, J., Sun, L., Luo, X., Barbieri, D., de Bruijn, J. D., van Blitterswijk, C. A., et al. (2017b). Cells responding to surface structure of calcium phosphate ceramics for bone regeneration. J. Tissue Eng. Regen. Med. 11, 3273-3283. doi: 10.1002/ term. 2236

Zura, R., Xiong, Z., Einhorn, T., Watson, J. T., Ostrum, R. F., Prayson, M. J., et al. (2016). Epidemiology of fracture nonunion in 18 human bones. JAMA Surg. 151:e162775. doi: 10.1001/jamasurg.2016.2775
Conflict of Interest Statement: The University Zurich has filed a patent on the microarchitecture of osteoconductive bone substitute with FW listed as sole inventor.

The remaining author declares that the research was conducted in the absence of any commercial or financial relationships that could be construed as a potential conflict of interest.

The reviewer VT and handling Editor declared their shared affiliation.

Copyright () 2018 Ghayor and Weber. This is an open-access article distributed under the terms of the Creative Commons Attribution License (CC BY). The use, distribution or reproduction in other forums is permitted, provided the original author(s) and the copyright owner(s) are credited and that the original publication in this journal is cited, in accordance with accepted academic practice. No use, distribution or reproduction is permitted which does not comply with these terms. 